\title{
Clinical significance of novel costimulatory molecule B7-H6 in human breast cancer
}

\author{
JING SUN ${ }^{1}$, HONG TAO $^{1}$, XIAONING LI ${ }^{1}$, LU WANG $^{2}$, JIE YANG ${ }^{1}$, \\ PINGPING WU $^{1}$, YAQIN ZHANG ${ }^{1}$ and YUNDI GUO ${ }^{1}$ \\ ${ }^{1}$ Institute of Medical Biotechnology, Suzhou Vocational Health College, Suzhou, Jiangsu 215009; \\ ${ }^{2}$ Department of Pathology, The Fourth Affiliated Hospital of Nanjing Medical University, Suzhou, Jiangsu 214062, P.R. China
}

Received January 9, 2016; Accepted January 12, 2017

DOI: $10.3892 / \mathrm{ol} .2017 .6417$

\begin{abstract}
B7 homolog 6 (B7-H6), a member of the B7 family, is as a cell-surface ligand for natural cytotoxicity triggering receptor 3 , which is expressed on natural killer cells. It has previously been reported that B7-H6 is undetectable in normal human tissues but is expressed on tumor cells. However, there are few studies focusing on the clinical significance of B7-H6 expression in human carcinoma, with the exception of three studies on ovarian, lung and gastric cancer. The present study investigated the expression of B7-H6 protein in pathologic tissue samples from 305 patients with breast cancer using immunohistochemistry. A high B7-H6 expression level was identified in tissues from $32.13 \%$ of patients with breast cancer. These patients were revealed to also exhibit a high expression level of human epidermal growth factor receptor 2, a shorter survival time and a higher rate of lymph node metastasis. Furthermore, the expression level of B7-H6 was not associated with patient age, breast cancer subtype, tumor size, tumor location or estrogen receptor expression. The results of the present study revealed that higher B7-H6 expression level in breast cancer tissues was positively associated with tumor progression. This indicates that B7-H6 is associated with the progression and immunoevasion of human breast cancer; however, the molecular mechanisms underlying this potential effect require further investigation.
\end{abstract}

\section{Introduction}

B7 homolog 6 (B7-H6), a novel member of the B7 family, was identified on tumor cell surfaces in 2009 (1). B7-H6 has sequence homology with other B7 molecules and similar to

Correspondence to: Professor Yundi Guo or Professor Jing Sun, Institute of Medical Biotechnology, Suzhou Vocational Health College, 28 Kehua Road, Suzhou, Jiangsu 215009, P.R. China

E-mail: gyd1964@163.com

E-mail: jsun@szhct.edu.cn

Key words: human breast cancer, B7 homolog 6, costimulatory molecule, natural cytotoxicity triggering receptor 3 other members of the B7 family, B7-H6 contains two extracellular immunoglobulin (Ig) domains; however, the receptor for B7-H6, located on natural killer (NK) cells, was not consistent with other B7 family member receptors, which are located on activated T cells. The receptor for B7-H6 is natural cytotoxicity triggering receptor 3 (NKp30) (1-3). NK cells are large granular lymphocytes that produce chemokines and cytokines, and participate in the inflammatory and adaptive immune response (4). NK cells are also an important part of the innate immune system as they directly kill transformed and virally infected cells (5).

B7 family members serve an essential role in regulating the immune response against transformed cells through a variety of mechanisms (6). As specific niches of B7 family members continue to be dissected, their diagnostic and therapeutic potential in tumors is becoming more apparent $(6,7)$, and this was highlighted in 2011 by the US Food and Drug Administration approval of an antibody targeting programmed death-ligand 1 (PD-L1) and cytotoxic T-lymphocyte-associated protein 4 in cancer $(8,9)$. The ability to successfully target cell cycle checkpoint regulators has since led to multiple clinical trials investigating antibodies targeting the signaling pathway that the B7 family participate in (10).

B7-H6 binds to NKp30, triggering antitumor NK cell cytotoxicity and cytokine secretion, thus B7-H6 functions as a tumor-induced self-molecule that alerts the innate immune system to cellular transformation $(11,12)$. Previous studies have investigated the association between B7-H6 expression and numerous types of tumor, including 65 cases of lung cancer, 60 cases of gastric cancer and 110 cases of human ovarian cancer (12-14). To the best of our knowledge, no previous studies have investigated the clinical significance of B7-H6 protein expression in patients with breast cancer.

The present study aimed to investigate the expression of B7-H6 protein in primary breast cancer by immunohistochemistry (IHC), and to identify the association between B7-H6 expression and the clinicopathological features and survival time of patients with breast cancer. The present study will aid in future studies examining the function of B7-H6 in the tumor immune response. The results of the present study also suggest that B7-H6 may have prognostic and therapeutic value in breast cancer. 


\section{Materials and methods}

Patients. Cancer tissues from 305 patients, who underwent surgery for breast cancer between January 2009 and March 2014 at the Department of General Surgery of The Fourth Hospital of Suzhou (Suzhou, China) and Shanghai Renji Hospital (Shanghai, China), were used in the present study. Patients who had received any preoperative chemotherapy or radiotherapy prior to surgery were excluded from the study. A total of 74 patients from this cohort were treated at the Department of General Surgery of The Fourth Hospital of Suzhou between January 2009 and December 2009. The other 231 patients were treated at the Department of General Surgery of Shanghai Renji Hospital between April 2010 and March 2014. The 305 tissue samples were stained with hematoxylin and eosin and final pathological diagnoses were confirmed. The patients' pathological reports were recorded, and their clinicopathological characteristics are presented in Table I. Survival data were collected from patient follow-ups. The present study was approved by the Ethics Review Board of The Fourth Hospital of Suzhou and Shanghai Renji Hospital. Written informed consent was obtained from all patients prior to enrollment in the present study.

Construction of the tissue microarray (TMA). A TMA containing a total of 305 tissue samples was prepared, with core tissue sections (diameter, $1.6 \mathrm{~mm}$ ) obtained from individual paraffin-embedded breast tumor samples (donor blocks) using a trephine. Sections (4- $\mu$ m-thick) were cut from each tissue sample, deparaffinized and dehydrated for IHC staining using the procedure described by Chen et al (15).

IHC staining. Rabbit anti-human $\mathrm{B} 7-\mathrm{H} 6$ polyclonal antibody was purchased from Abcam (Cambridge, UK; cat. no. Ab121794) and was diluted 1:40 to produce a working concentration of $7.5 \mu \mathrm{g} / \mathrm{ml}$. Mouse anti-human cluster of differentiation (CD) 56, a marker of NK cells, antibody was purchased from Fuzhou Maixin Biotech Co., Ltd. (Fuzhou, China; cat. no. Kit-0028). The horseradish peroxidase (HRP)-conjugated anti-mouse/rabbit secondary antibodies kit was also purchased from Fuzhou Maixin Biotech Co.,Ltd. (cat. no. kit-5010) and the IHC staining for B7-H6 and CD56 was performed according to the manufacturer's protocol. Breast cancer TMA sections were incubated with Mayer's hematoxylin solution, and incubated at $100^{\circ} \mathrm{C}$ for $30 \mathrm{~min}$ in citrate solutions. The tissue sections were incubated at $4^{\circ} \mathrm{C}$ for $16 \mathrm{~h}$ with anti-B7-H6 and anti-CD56 antibodies and further incubated at room temperature for $15 \mathrm{~min}$ with the HRP-conjugated secondary antibodies. Unrelated isotype-matched rabbit IgG Ab-1 antibody (cat. no. NC-100, Fuzhou Maixin Biotech. Co., Ltd.) and mouse IgG Ab-1 antibody (cat. no. NC-748, Fuzhou Maxin Biotech. Co., Ltd.) were used as the control for nonspecific staining.

Evaluation of IHC staining. Two independent observers who were blinded to the clinicopathological characteristics of the patients examined the immunostained sections. B7-H6 expression level analysis was limited to tumors with a sufficient amount of tissue at a certain stage of cancer development for IHC evaluation. A semiquantitative scoring system based on the amount of positively stained tumor cells and the staining intensity was used to evaluate the expression level of B7-H6 protein. An estimate of the proportion of positively stained cells of $0-10 \%$ scored $1,11-30 \%$ scored $2,31-60 \%$ scored 3 , and $61-100 \%$ scored 4 . An intensity factor ranged from 1 (weak positive, staining intensity marginally exceeding the background) to 4 (strong positive, dark brown staining clearly visible on macroscopic inspection of the slide). The immunostaining scoring was conducted by multiplying the scores for the proportion and the intensity of positive-staining cells. Scores between 1 and 8 were defined as the low expression group, and scores between 9 and 16 were defined as the high expression group.

Statistical analysis. SPSS (version 17.0; SPSS, Inc., Chicago, IL, USA) was used for statistic analysis. All data are presented as the mean \pm standard deviation. Statistical analysis was performed using the Student's $t$-test and analysis of one-way analysis of variance. Correlations were evaluated with Pearson's correlation coefficient. $\mathrm{P}<0.05$ was considered to indicate a statistically significant difference. Kaplan-Meier estimator survival plots were generated, and comparisons between the survival curves were performed using a log-rank test.

\section{Results}

B7-H6 protein expression in breast cancer tissues. In order to determine the expression levels of B7-H6 protein in breast cancer tissues, IHC analysis was performed (Fig. 1). This revealed that B7-H6 was present in 216/305 (70.82\%) of the breast cancer tissues. B7-H6 was predominantly localized in the membrane and cytoplasm of breasts tumor cells. In order to investigate the association between the clinicopathological characteristics of breast cancer and B7-H6 protein expression, the 305 patients were divided into two major subgroups according to their amount of positively stained cells and intensity of B7-H6 staining as follows: Low B7-H6 expression $(\mathrm{n}=206)$; and high B7-H6 expression $(\mathrm{n}=99)$.

Association between B7-H6 expression and the clinicopathological characteristics of patients with breast cancer. The association between the clinicopathological characteristics of patients with breast cancer and B7-H6 expression are presented in Table I. This revealed that B7-H6 expression was significantly correlated with nodal metastasis $(\mathrm{P}=0.003)$; however, it was not associated with the other clinicopathological parameters, including age, tumor location and tumor size. Furthermore, B7-H6 expression was significantly positively correlated with human epidermal growth factor receptor 2 (HER2) expression status $(\mathrm{P}<0.0001)$, a marker of metastasis and predictor of poor prognosis in breast cancer. B7-H6 expression was significantly negatively correlated with progesterone receptor (PR) expression status $(\mathrm{P}=0.0392)$, a marker of good prognosis in breast cancer. These results suggest that patients with breast cancer who have higher B7-H6 expression levels have a higher risk of metastases and a poorer prognosis.

Association between B7-H6 expression and post-operative prognosis in patients with breast cancer. In order to further investigate the prognostic value of B7-H6 expression in human 
Table I. Association between B7-H6 protein expression and the clinicopathological characteristics of patients with breast cancer.

\begin{tabular}{|c|c|c|c|}
\hline \multirow[b]{2}{*}{$\begin{array}{l}\text { Clinicopathological } \\
\text { characteristic }\end{array}$} & \multicolumn{2}{|c|}{ Percentage of patients } & \multirow[b]{2}{*}{ P-value } \\
\hline & $\begin{array}{c}\text { Low } \\
\text { expression } \\
\text { of B7-H6 }\end{array}$ & $\begin{array}{c}\text { High } \\
\text { expression } \\
\text { of B7-H6 }\end{array}$ & \\
\hline Breast cancer subtype & & & 0.264 \\
\hline Lumin A & 14 & 10 & \\
\hline Lumin B & 61 & 34 & \\
\hline $\mathrm{HER}^{+}$ & 58 & 31 & \\
\hline Basal-like & 12 & 1 & \\
\hline Normal-like & 4 & 1 & \\
\hline Tumor location & & & 0.254 \\
\hline Left breast & 101 & 38 & \\
\hline Right breast & 101 & 56 & \\
\hline Bitemporal breast & 5 & 4 & \\
\hline Tumor size, $\mathrm{cm}$ & & & 0.8243 \\
\hline$<2.5$ & 110 & 45 & \\
\hline$\geq 2.5$ & 97 & 42 & \\
\hline Lymph node metastasis & & & $0.003^{\mathrm{a}}$ \\
\hline Absent & 87 & 25 & \\
\hline Present & 65 & 45 & \\
\hline HER2 expression status & & & $0.0001^{\mathrm{a}}$ \\
\hline Negative & 74 & 22 & \\
\hline Weak & 42 & 12 & \\
\hline Medium & 43 & 14 & \\
\hline Strong & 36 & 36 & \\
\hline ER expression status & & & 0.159 \\
\hline Negative & 88 & 30 & \\
\hline Positive & 115 & 57 & \\
\hline PR expression status & & & $0.0392^{\mathrm{a}}$ \\
\hline Negative & 99 & 32 & \\
\hline Positive & 79 & 45 & \\
\hline
\end{tabular}

The median age of patients with low expression of B7-H6 was 55.11 years (range, 26-94 years). The median age of patients with high expression of B7-H6 was 55.22 years (range, 37-70 years). A $\chi^{2}$ test was used to analyze whether the breast cancer subtype, tumor location, tumor size, lymph node metastasis and HER2, ER and PR expression were associated with B7-H6 expression. ${ }^{\mathrm{a}} \mathrm{P}<0.005$. B7-H6, $\mathrm{B} 7$ homolog 6; ER, estrogen receptor; PR, progesterone receptor; HER2, human epidermal growth factor receptor.

breast cancer, a log-rank test was performed. A total of 51 patient overall survival rates and cumulative survival rate were determined using the Kaplan-Meier estimator method and analyzed using a log-rank test. The survival analysis demonstrated that the overall survival rate of the patient subgroup with low B7-H6 expression was significantly higher compared with the subgroup with high $\mathrm{B} 7-\mathrm{H} 6$ expression $(\mathrm{P}=0.0394$; hazard ratio, $0.1941,95 \%$ confidence interval, 0.04076-0.9238; Fig. 2).

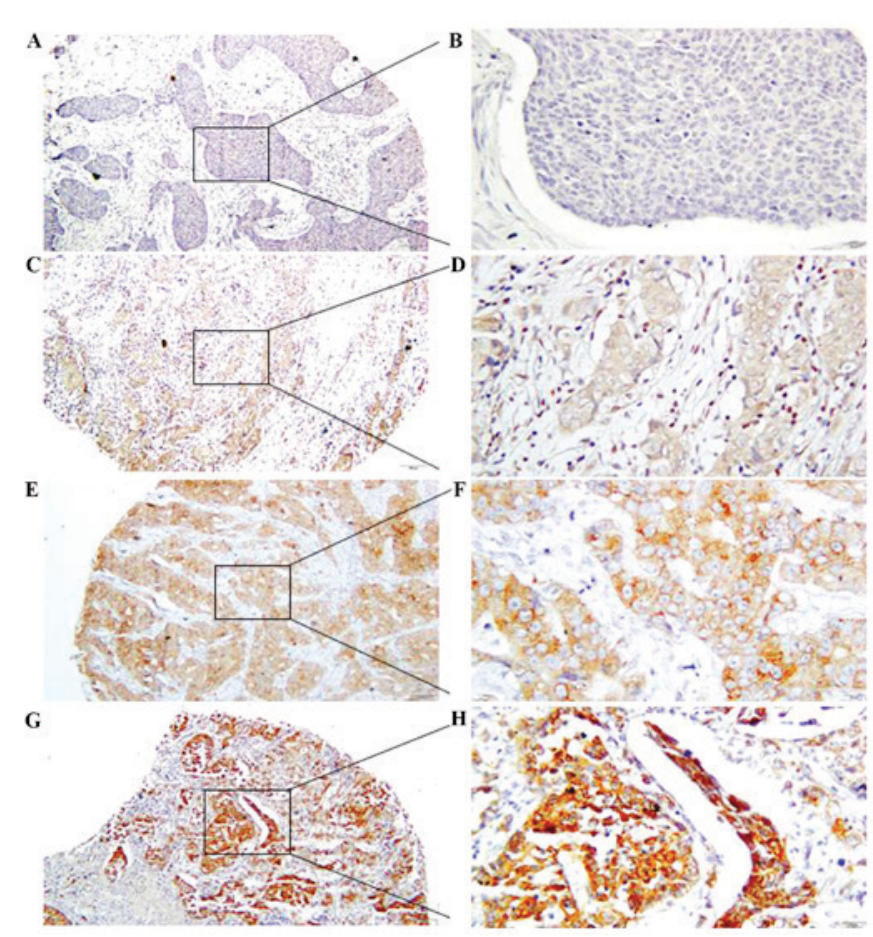

Figure 1. Representative immunohistochemical staining of B7 homolog 6 expression in human breast cancer. Negative staining at a magnification of (A) $\times 100$ and (B) x400. Weak positive staining at a magnification of (C) x100 and (D) x400. Moderate positive staining at a magnification of (E) x100 and (F) $x 400$. Strong positive staining at a magnification of $(\mathrm{G}) \times 100$ and $(\mathrm{H})$ $\mathrm{x} 400$.

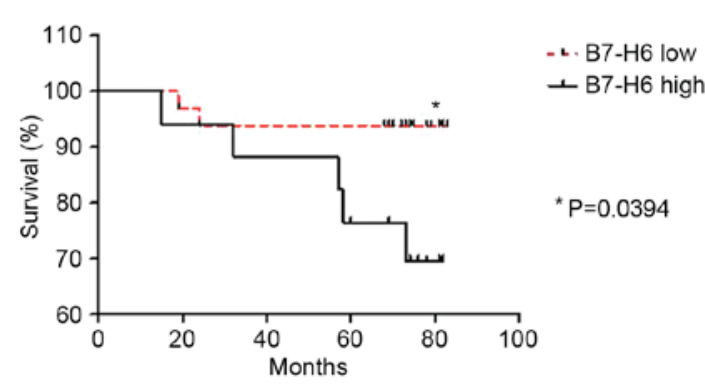

Figure 2. Kaplan-Meier estimator survival analysis for the association between overall survival rate and the expression level of B7-H6 in patients with breast cancer. ${ }^{*} \mathrm{P}=0.0394$. B7-H6, B7 homolog 6 .

\section{Discussion}

The B7 family, and their CD28 receptor family of costimulatory and coinhibitory molecules, have previously been demonstrated to serve a potential role in the immune response, and these molecules have been revealed to be effective diagnostic markers and therapeutic targets for tumors $(16,17)$. This was highlighted by the approval of the drug ipilimumab and ongoing clinical trials of treatments targeting the PD-L1 signaling pathway (18). B7-H6, a novel B7 family member, acts as a co-stimulatory ligand that delivers a stimulatory signal to NK cells through its receptor NKp30 (1). The present study aimed to investigate the prognostic value of B7-H6 expression in breast cancer and its potential therapeutic role in tumor adoptive immunotherapy, using TMAs and IHC. 
It has previously been reported that B7-H6 mRNA was undetectable in a tissue array of 65 normal adult tissues (1), consistent with the absence of the protein on circulating cells isolated from healthy individuals (19). In contrast, B7-H6 cell surface expression has been observed in cell lines from various tumor types, including lymphoma, leukemia and melanoma $(3,14,20)$. In addition, B7-H6 expression has been detected in tumor tissues, such as gastrointestinal, lung and ovarian $(1,12-14,21)$. The results of the present study demonstrated that B7-H6 was predominantly localized to the membrane of breast cancer cells in 216/305 (70.82\%) of the patients. The pattern of B7-H6 expression, which appears to be limited to tumor cells, is frequently regarded as an example of stress-induced self-recognition by NK cells.

B7-H6 was revealed to be a ligand of NKp30 on NK cells, which delivers an activating signal into NK cells (1). NKp30 consists of a single $\operatorname{IgV}$ domain in its extracellular region, a unique structural feature of the CD28 family (22). Another ligand of NKp30 is HLA-B associated transcript, which is a nuclear protein involved in the tumor protein p53 signaling pathway and apoptosis induction following DNA damage-induced cellular stress $(23,24)$. NKp30 was previously revealed to mediate antitumor effects in gastrointestinal stromal tumors and lymphoid leukemia $(25,26)$. Numerous previous studies have indicated that the B7-H6-NKp30 signaling pathway is implicated in antitumor activity by stimulating primary NK cells to produce interleukin-2 and interferon- $\gamma($ IFN- $\gamma)$, thus enhancing cytotoxic activity $(1,21)$.

The present study investigated the theory that B7-H6 is able to facilitate the elimination of tumor cells by interacting with its receptor NKp30, thus serving a role in antitumor immunity. Previous in vitro studies have also suggested that B7-H6 may be useful for treatment to target tumor cells expressing B7-H6 $(3,19,27)$. Incubation of lymphoma cells with a fusion protein containing B7-H6 and 7D8, an antibody that recognizes CD20, enhanced NK cell activation and cytotoxicity in vitro (28). In the present study, the analysis of 305 tissue samples from patients with breast cancer did not suggest that B7-H6 has an antitumor function. However, the significant positive correlation identified between B7-H6 expression and lymph node metastasis indicates that B7-H6 may participate in tumor progression and development. Furthermore, a significant positive correlation was identified between B7-H6 protein expression and HER2 expression status, an important maker of poor breast cancer prognosis, recurrence and metastasis.

Similarly to other B7 family members, B7-H6 may exist as a surface/cytosolic molecule in tumor cells and as a soluble molecule in the peritoneal fluid (21). The soluble from of B7-H6 has been detected in the serum of patients with malignant melanoma, neuroblastoma and ovarian carcinoma $(3,27,29)$. Previous studies have identified elevated levels of soluble B7-H6 in the blood sera of patients with gram-negative sepsis compared with healthy control individuals $(23,24)$. Furthermore, the serum concentration of soluble B7-H6, which was detected by disintegrin and metalloprotease (ADAM)-10 and ADAM-17, was associated with the downregulation of NKp30 and inhibited NK cell functions in vitro by impairing IFN- $\gamma$ production $(3,29)$. This indicates that high expression levels of B7-H6 in breast cancer tissues may contribute to cancer cell immunoevasion.
The present study, to the best of our knowledge, demonstrated for the first time the clinical significance of B7-H6 expression in breast cancer, and revealed that B7-H6 protein expression was positively associated with breast cancer metastasis, supporting the hypothesis that B7-H6 expression is involved in the progression of human breast cancer. The present study revealed the possibility that B7-H6 serves a role in tumor immunoevasion and may be a potential target for tumor therapy in the future.

\section{Acknowledgements}

The present study was supported by the Jiangsu Province University Outstanding Science and Technology Innovation Team (grant no. 2015023) and the Qinglan Project (2013).

\section{References}

1. BrandtCS, Baratin M, Yi EC, Kennedy J, Gao Z, Fox B, Haldeman B, Ostrander CD, Kaifu T, Chabannon C, et al: The B7 family member B7-H6 is a tumor cell ligand for the activating natural killer cell receptor NKp30 in humans. J Exp Med 206: 1495-1503, 2009.

2. Xu X, Narni-Mancinelli E, Cantoni C, Li Y, Guia S, Gauthier L, Chen Q, Moretta A, Vély F, Eisenstein E, et al: Structural insights into the inhibitory mechanism of an Antibody against B7-H6, a stress-induced cellular ligand for the natural killer cell receptor NKp30. J Mol Biol 428: 4457-4466, 2016.

3. Pesce S, Tabellini G, Cantoni C, Patrizi O, Coltrini D, Rampinelli F, Matta J, Vivier E, Moretta A, Parolini S and Marcenaro E: B7-H6-mediated downregulation of NKp30 in NK cells contributes to ovarian carcinoma immune escape. Oncoimmunology 4: e1001224, 2015.

4. Vivier E, Tomasello E, Baratin M, WalzerT and Ugolini S: Functions of natural killer cells. Nat Immunol 9: 503-510, 2008.

5. Bretscher P and Cohn M: A theory of self-nonself discrimination. Science 169: 1042-1049, 1970.

6. Flies DB and Chen L: The new B7s: Playing a pivotal role in tumor immunity. J Immunother 30: 251-260, 2007.

7. Hurwitz AA, Kwon ED and van Elsas A: Costimulatory wars: The tumor menace. Curr Opin Immunol 12: 589-596, 2000.

8. Mahoney KM and Atkins MB: Prognostic and predictive markers for the new immunotherapies. Oncology (Williston Park) 28 (Suppl 3): S39-S48, 2016.

9. Mahoney KM, Freeman GJ and McDermott DF: The next immune-checkpoint inhibitors: PD-1/PD-L1 blockade in melanoma. Clin Ther 37: 764-782, 2015.

10. Ceeraz S, Nowak EC and Noelle RJ: B7 family checkpoint regulators in immune regulation and disease. Trends Immunol 34: 556-563, 2013.

11. Matta J, Baratin M, Chiche L, Forel JM, Cognet C, Thomas G, Farnarier C, Piperoglou C, Papazian L, Chaussabel D, et al: Induction of B7-H6, a ligand for the natural killer cell-activating receptor NKp30, in inflammatory conditions. Blood 122: 394-404, 2013.

12. Zhang X, Zhang G, Qin Y, Bai R and Huang J: B7-H6 expression in non-small cell lung cancers. Int J Clin Exp Pathol 7: 6936-6942, 2014.

13. Chen XJ, Shen J, Zhang GB and Chen WC: B7-H6 protein expression has no prognostic significance in human gastric carcinoma. Pathol Oncol Res 20: 203-207, 2014.

14. Zhou Y, Xu Y, Chen L, Xu B, Wu C and Jiang J: B7-H6 expression correlates with cancer progression and patient's survival in human ovarian cancer. Int J Clin Exp Pathol 8: 9428-9433, 2015.

15. Chen L, Deng H, Lu M, Xu B, Wang Q, Jiang J and Wu C: B7-H1 expression associates with tumor invasion and predicts patient's survival in human esophageal cancer. Int J Clin Exp Pathol 7: 6015-6023, 2014.

16. Seliger B and Quandt D: The expression, function, and clinical relevance of B7 family members in cancer. Cancer Immunol Immunother 61: 1327-1341, 2012.

17. Lu B, Chen L, Liu L, Zhu Y, Wu C, Jiang J and Zhang X: T-cell-mediated tumor immune surveillance and expression of $\mathrm{B} 7$ co-inhibitory molecules in cancers of the upper gastrointestinal tract. Immunol Res 50: 269-275, 2011. 
18. Topalian SL, Hodi FS, Brahmer JR, Gettinger SN, Smith DC, McDermott DF, Powderly JD, Carvajal RD, Sosman JA, Atkins MB, et al: Safety, activity and immune correlates of anti-PD-1 antibody in cancer. N Engl J Med 366: 2443-2454, 2012.

19. Kaifu T, Escalière B, Gastinel LN, Vivier E and Baratin M: B7-H6/NKp30 interaction: A mechanism of alerting NK cells against tumors. Cell Mol Life Sci 68: 3531-3539, 2011.

20. Guo JG, Guo CC, He ZQ, Liu ZG, Wang Y and Mou YG: Clinical significance of B7-H6 protein expression in astrocytoma. Onco Targets Ther 9: 3291-3297, 2016.

21. Fiegler N, Textor S, Arnold A, Rölle A, Oehme I, Breuhahn K, Moldenhauer G, Witzens-Harig $M$ and Cerwenka A: Downregulation of the activating NKp30 ligand B7-H6 by HDAC inhibitors impairs tumor cell recognition by NK cells. Blood 122: 684-693, 2013.

22. Joyce MG, Tran P, Zhuravleva MA, Jaw J, Colonna M and Sun PD: Crystal structure of human natural cytotoxicity receptor NKp30 and identification of its ligand binding site. Proc Natl Acad Sci USA 108: 6223-6228, 2011.

23. Pogge von Strandmann E, Simhadri VR, von Tresckow B, Sasse S, Reiners KS, Hansen HP, Rothe A, Böll B, Simhadri VL Borchmann P, et al: Human leukocyte antigen-B-associated transcript 3 is released from tumor cells and engages the NKp30 receptor on natural killer cells. Immunity 27: 965-974, 2007.

24. Sasaki T, Gan EC, Wakeham A, Kornbluth S, Mak TW and Okada H: HLA-B-associated transcript 3 (Bat3)/Scythe is essential for p300-mediated acetylation of p53. Genes Dev 21: $848-861,2007$.
25. Correia DV, Fogli M, Hudspeth K, da Silva MG, Mavilio D and Silva-Santos B: Differentiation of human peripheral blood V811+ $\mathrm{T}$ cells expressing the natural cytotoxicity receptor NKp30 for recognition of lymphoid leukemia cells. Blood 118: 992-1001, 2011 .

26. Delahaye NF, Rusakiewicz S, Martins I, Ménard C, Roux S, Lyonnet L, Paul P, Sarabi M, Chaput N, Semeraro M, et al: Alternatively spliced NKp30 isoforms affect the prognosis of gastrointestinal stromal tumors. Nat Med 17: 700-707, 2011.

27. Schlecker E, Fiegler N, Arnold A, Altevogt P, Rose-John S, Moldenhauer G, Sucker A, Paschen A, von Strandmann EP, Textor S and Cerwenka A: Metalloprotease-mediated tumor cell shedding of B7-H6, the ligand of the natural killer cell-activating receptor NKp30. Cancer Res 74: 3429-3440, 2014.

28. Kellner C, Maurer T, Hallack D, Repp R, van de Winkel JG, Parren PW, Valerius T, Humpe A, Gramatzki M and Peipp M: Mimicking an induced self phenotype by coating lymphomas with the NKp30 ligand B7-H6 promotes NK cell cytotoxicity. J Immunol 189: 5037-5046, 2012.

29. Semeraro M, Rusakiewicz S, Minard-Colin V, Delahaye NF Enot D, Vély F, Marabelle A, Papoular B, Piperoglou C, Ponzoni M, et al: Clinical impact of the NKp30/B7-H6 axis in high-risk neuroblastoma patients. Sci Transl Med 7: 283ra255, 2015. 